\title{
¿Aprender español de las telenovelas?: un aporte desde la perspectiva de receptores rumanos $^{1}$
}

\author{
Silvia Rivera Alfaro \\ Programa de Español como Lengua Extranjera \\ Universidad de Costa Rica
}

\begin{abstract}
Resumen
Este trabajo explora la utilidad de las telenovelas como producto cultural para la difusión del español -en especial su variante americana- desde la perspectiva de consumidores de nacionalidad rumana. Para el análisis, se toman en cuenta las nociones de globalización lingüística (López Morales, 2006), el concepto de lenguaje (Rossi-Landi, 1970) y de mercado lingüístico (Bourdieu, 2001). Entre los resultados sobresale que estos seriales llevan a las personas rumanas que los consumen a convertirse en aprendientes involuntarios, que la lengua aprendida es útil para quienes son televidentes en situaciones prácticas y que las telenovelas permiten colocar el español latinoamericano y sus variedades dentro del mercado lingüístico global.
\end{abstract}

Palabras claves: telenovelas, globalización lingüística, español como lengua extranjera, español de América, Rumanía

\section{Abstract}

This work explores usefulness of 'telenovelas' as a cultural product for the diffusion of Spansih - specially its American variant-from the perspective of the Romanian consumers. For the analysis, we take into account the notions of linguistic globalization (López Morales, 2006), the concept of language (Rossi-Landi, 1970), and linguistic market (Bourdieu, 2001). Among the results, it is highligthed that these shows conduct Romanians who consum them to become involuntary language learners, that the learned language is useful for the TV followers in practical situations, 
and that telefilms allow positioning American Spanish and its varieties in the global linguistic market.

Key words: telenovelas, linguistic globalization, Spanish as a foreign language, ELE, American Spanish, Romania

\section{Introducción}

$\mathrm{L}$ as telenovelas son un producto cultural creado originalmente en Latinoamérica que ha llegado a tener una exportación mundial masiva (Fuenzalida, 2005; Salgueiro, 2004); como producto, tienen gran impacto en la globalización lingüística tanto en la región latinoamericana como en el nivel internacional (Covarrubias, 2010; López Morales, 2006). Dicho producto entró a países ex comunistas de Europa del Este desde los años noventa; su función fue llenar las franjas de emisiones de ficción, que no podían ser producidas por su costo económico (Mazziotti, 2006, p.61). Hasta el presente, en algunos lugares, las telenovelas se transmiten dobladas a la lengua local; en otros, como en Rumanía, se transmiten únicamente con subtítulos.

Estas series televisivas han sido abordadas desde diversas disciplinas; sin embargo, escasamente desde la lingüística. Además, en este campo, son prácticamente nulas las investigaciones que presenten la perspectiva de receptores del show en relación con la lengua. En los trabajos lingüísticos, se discute su papel para la difusión del español, tanto en comunidades hispanohablantes (Fuentes, 2001; López Morales, 2006) como en otras latitudes (López Morales, 2006 y Covarrubias, 2010) ${ }^{2}$. Para Rumanía, Jimeno Panés (2006) menciona, con respecto a la enseñanza no reglada de Español como Lengua Extranjera (ELE), que el interés por el idioma ha crecido gracias a las telenovelas, entre otros factores (p.304-305) ${ }^{3}$; se trata de un fenómeno que también se evidencia en los medios de comunicación (Tucan, 2007; EFE, 2009; Opronoiu, 2012).

Así las cosas, el objetivo de este trabajo es explorar la utilidad de las telenovelas como producto cultural para la difusión del español ${ }^{4}$-en especial, del español de América $^{5}$ - a partir de la perspectiva de personas rumanas que las consumen. Su importancia ${ }^{6}$ radica en la escasez de investigaciones que den razón de la relación de la lengua con los productos culturales latinoamericanos en distintas latitudes; conocer esta relación desde el punto de vista de quienes son receptores es de especial relevancia, pues son estas personas quienes permiten que estos productos continúen circulando en el mercado $\mathrm{y}$, gracias a ellos, la lengua española.

\section{Metodología}

Para la recolección de datos se crearon dos instrumentos. Estos fueron validados por la Dra. Carla Jara Murillo, del Departamento de Lingüística de la Universidad de Costa Rica. Ambos instrumentos se publicaron en línea utilizando Google Docs y, por este medio, se convirtieron en encuestas semicerradas (independientes una de la otra) disponibles en línea. 
Por medio de la primera encuesta, se consiguió de fuente primaria la opinión de 25 personas rumanas que sean o hayan sido receptoras de dicho programa. $\mathrm{Su}$ opinión se complementó con datos de observadores externos familiarizados con la cultura rumana. Con este fin, la segunda encuesta se dirigió a personas latinoamericanas que hubieran vivido en Rumanía por un período mayor a seis meses durante los últimos 5 años al momento de ser encuestadas (abril, 2013); en este proceso, se contó con 5 participantes.

El análisis se realizó primero comparando pregunta a pregunta la información suministrada, por un lado, por las personas rumanas ${ }^{7} \mathrm{y}$, por otro, la de las personas hablantes de español. Luego, se complementaron los datos de quienes son o han sido receptores de telenovelas con los de las personas hablantes nativas de español.

\section{Perspectiva teórica}

En este trabajo se entiende el lenguaje como un instrumento de naturaleza social que permite satisfacer necesidades sociales y se utiliza al hablar (una actividad social) para hacer un trabajo y crear palabras/mensajes, que son productos de una comunidad específica (Rossi-Landi, 1970) y pueden ser consumidos (Ponzio, 2008). Socialmente, el lenguaje implica un capital simbólico (Bourdieu, 2001) que existe en un mercado lingüístico: "Lo que circula en el mercado lingüístico no es "la lengua», sino discursos estilísticamente caracterizados, discursos que se colocan a la vez del lado de la producción..." (Bourdieu, 2001, p.13).

En ese mercado lingüístico se da la globalización lingüística, que es definida por López Morales (2006) como: "1. Difusión mundial de una lengua que fomenta la uniformidad lingüística más allá de sus fronteras nacionales. 2. Difusión internacional de una variedad dialectal específica de una lengua natural que fomenta la uniformidad lingüística en todo su ámbito idiomático" (p.24). Agregamos a estos conceptos el de: difusión internacional de una lengua más allá de su ámbito idiomático que puede promover el conocimiento -al menos básico- de dicha lengua, o al menos unas actitudes lingüísticas hacia ella o una de sus variedades en una comunidad consumidora de productos culturales que incluyen productos lingüísticos.

Las telenovelas son productos culturales audiovisuales que contribuyen a la globalización lingüística (López Morales, 2006). Narran la historia de unos personajes que “... enormes audiencias de todas las clases sociales a lo largo de todo el mundo siguen [...] día a día, durante meses" (Mazziotti, 2006, p.21); es decir, incluyen palabras/mensajes trasmitidos con una frecuencia determinada.

En estos seriales el lenguaje es planificado por medio de un guion, pues debe facilitarse su comprensión para la exportación masiva. Por esta razón, "se reducen del todo, o al mínimo, los localismos léxicos y fraseológicos" (López Morales, 2006, p.159). Los productos lingüísticos del guion son concretados por actores que tienen la potestad de realizar este trabajo simbólico (Bernárdez, 2008, p. 229) al asumir personajes. Los actores, según cuenta López Morales (2006), incluso son preparados por profesionales para acercarse más a la variedad dialectal del lugar donde se produce la telenovela.

Los consumidores de esos mensajes orales son los receptores, 
que aproximadamente suman dos mil millones (Mazziotti, 2006, p.64). Ellos podrían devenir aprendientes de español en el proceso de consumo de las telenovelas o por influencia de estas, pues el aprendizaje de segundas lenguas engloba "procesos conscientes $e$ inconscientes [cursiva añadida] mediante los cuales el aprendiente alcanza un determinado nivel de competencia en una lengua segunda" (Centro Virtual Cervantes, s.f., párr.1).

Se considera posible relacionar los conceptos arriba expuestos y afirmar que aprender una lengua implica "adquirir un producto lingüístico" consciente o inconscientemente: "exige una inversión muy considerable: hay que dedicar tiempo y dinero [...] y una actualización permanente" (Bernárdez, 2008, p.231-232). Aprender una lengua permite entrar en un mercado lingüístico con un determinado capital simbólico que dependería de cómo es vista la lengua que se adquiere.

\section{Análisis y resultados}

El análisis que se presenta a continuación se ordena pregunta a pregunta tal y como se ha organizado el cuestionario. Se inicia analizando cuál es el español que se consume; luego, se pasa del consumo al aprendizaje. Después, se aborda el tema de la lengua española y sus variedades. Finalmente, se llega al tema de la lengua como producto.

\section{¿Qué español se consume?}

Partir de la lengua como producto conlleva a observar los países de procedencia de las telenovelas consumidas por la comunidad rumana. La Tabla 1 evidencia que la mayoría proviene de América Latina; España es mencionada una única vez.

Tabla 1. Telenovelas por país

\begin{tabular}{|c|c|c|c|}
\hline & \multicolumn{2}{|c|}{ Frecuencia } & \multirow[b]{2}{*}{ Nombre de la telenovela (si fue indicado) } \\
\hline & $\begin{array}{c}\text { N. }{ }^{\circ} \\
\text { personas }\end{array}$ & Porcentaje & \\
\hline México & 19 & 35,19 & $\begin{array}{l}\text { Marimar (3), María la del barrio (2), Rosalinda (2), } \\
\text { María Mercedes (2), Rebelde, Salomé (2), Primer } \\
\text { amor, Rubí (2), Carita de Ángel, Amor Real, Cuan- } \\
\text { do seas mía, Paloma }\end{array}$ \\
\hline Argentina & 13 & 24,07 & Muñeca Brava (2) \\
\hline Colombia & 8 & 14,81 & Betty la fea, Pasión de Gavilanes (2) \\
\hline Venezuela & 8 & 14,81 & - \\
\hline España & 3 & 5,56 & - \\
\hline Uruguay & 1 & 1,85 & - \\
\hline Cuba & 1 & 1,85 & - \\
\hline Miami & 1 & 1,85 & Prisionera \\
\hline Total & 54 & $100 \%$ & - \\
\hline
\end{tabular}


Se debe resaltar que las personas receptoras no siempre conocen con claridad el origen de la telenovela; por ejemplo, una de las menciones de Pasión de Gavilanes la atribuye a la industria mexicana. En algunos casos, se conoce únicamente el país o el nombre. Por ejemplo, Miami fue agregado a partir del nombre de la telenovela, único dato obtenido.

\section{Del consumo al aprendizaje}

Desde la perspectiva de estos rumanos y rumanas, sí es posible aprender español viendo telenovelas. De las 25 personas entrevistadas, 24 lo consideran posible e indican que han conocido a alguien que lo hizo.

$\mathrm{Al}$ preguntarles un número aproximado de conocidos y conocidas que aprendieron español de esta manera, la respuesta más frecuente fue 10 (indicada por 8); luego, 20 y 5 (indicados por 3 personas cada uno), seguida por 15, "3 a 4", 2 y 1 (indicadas por 2); y, 3 y 6 (indicadas también por 1). Visto de otra manera: estas 24 personas conocerían, en total, entre 206 y 208 personas que lo han hecho.

Se suma a esta la opinión de 4 de las 5 personas hablantes nativas de español, que coincidieron en que sí es posible y afirman haber conocido a 19,6 , 2 y de "1 a 5 " rumanos que lo hicieron. En total serían entre 28 y 32 personas.

Si tomamos los números aproximados totales de personas conocidas que aprendieron de las telenovelas y los dividimos entre la cantidad de informantes, tenemos un promedio bastante alto: cada persona de Rumanía conocería 8,5 u 8,6 que aprendieron de este modo y cada persona de Latinoamérica, 7 u 8.
Además, de las 24 personas rumanas, 21 indican haberlo aprendido ellas mismas. Según estas 21, el aprendizaje del español por medio de las telenovelas es un proceso: involuntario ("sin darme cuenta y sin querer aprender español"), fácil ("no hice nada en especial"), "natural", que se realiza a lo largo del tiempo viendo frecuentemente los episodios y escuchando activamente los diálogos mientras se leen los subtítulos por el gusto de ver las telenovelas o -incluso- sin estar atento ("nunca he visto de modo activo, [...] oía sin querer"). Según estos receptores, el aprendizaje puede llevarse a cabo de manera inconsciente ("no sabía que hablaba"), pues no se enteran de que están aprendiendo hasta descubrirlo en una situación social en la que requieren la lengua.

Considerando el proceso, resulta de particular interés qué aprenden por medio de las telenovelas, especialmente, cuáles son las primeras frases o palabras, pues por estas inicia el aprendizaje. Quienes completaron el instrumento indicaron 87 vocablos $y$ frases. De estos, los que aparecen con mayor frecuencia -individualmente- o dentro de una frase- son: hola y embarazada, mencionadas 7 veces cada una; te quiero, 6 veces; y te amo, 5 .

La Tabla 2 organiza las expresiones por categorías a partir de las funciones que puedan tener para la producción del mensaje de la telenovela. La grafía de las palabras se incluye tal y como fue escrita en el cuestionario (la "ch" debe leerse como "qu"); entre paréntesis, se indica el número de veces que se repiten las palabras: 
Tabla 2: Primeras palabras y frases aprendidas por los rumanos

\begin{tabular}{|c|c|c|}
\hline Ámbito & Palabra o frase & N. ${ }^{\circ}$ \\
\hline Saludos y despedidas & $\begin{array}{c}\text { Hola (6), ola, que tal (3), commo te llamas?, como estas? (3), } \\
\text { buneoz diaz, buenas días, buenos dia, adios }\end{array}$ & 18 \\
\hline Expresiones de afecto & $\begin{array}{l}\text { Mi amor (2), mi amore, te amo (5), te chero, te quero, te chero } \\
\text { mucho, beso, te quiero mucho }(2) \text {, te quiero, te amor }\end{array}$ & 15 \\
\hline $\begin{array}{l}\text { Familia y relaciones } \\
\text { sociales }\end{array}$ & $\begin{array}{c}\text { Padre (2), mi papa, ermano, hermano, hija, madre, novio, Luis } \\
\text { Fernando es tu hermano, seniora }\end{array}$ & 10 \\
\hline $\begin{array}{l}\text { Acciones en general y } \\
\text { otras frases complejas }\end{array}$ & $\begin{array}{l}\text { Cambia te, no tiengo, no lloraj, me gusta }(2) \text {, me gusta tu..., } \\
\text { estoi aprovechando de este tiempo, quisieras que..., con chen } \\
\text { esta ablando, es la misma muher, no me pasa }\end{array}$ & 9 \\
\hline $\begin{array}{l}\text { Expresiones de pelea, } \\
\text { desamor y sufrimiento }\end{array}$ & $\begin{array}{l}\text { No me enganas, te odio, malagradecida, mentirosa, desgra- } \\
\text { ciada, me duele, quel dolor, este no es amor }\end{array}$ & 8 \\
\hline Otros & $\begin{array}{c}\text { El caracol, la cucaracha, suerte, mochila, Ai caramba, comida, } \\
\text { por que, que ora es? }\end{array}$ & 8 \\
\hline Lugares & Pueblo, ciudad, cuarto, vamos a la playa & 4 \\
\hline "Estoy embarazada" & $\begin{array}{c}\text { (Io) Sono embarasada (2), estoy embarazada, embarasada, } \\
\text { estoi embarasada (2), embarazada }\end{array}$ & 7 \\
\hline Adverbios & Muchísimo, muy bien (2) & 3 \\
\hline Afirmaciones & Si!, claro que si (2) & 3 \\
\hline \multirow[t]{2}{*}{ Sin respuesta } & & 2 \\
\hline & Total & 87 \\
\hline
\end{tabular}

$\mathrm{Al}$ confrontar estos resultados con la opinión de latinoamericanos y latinoamericanas, se evidencia el protagonismo de los mismos ámbitos, pues todas sus frases pueden organizarse en las categorías más frecuentes de la tabla. Dos mencionaron "estoy embarazada"; las otras expresiones aparecen una vez: atrevido, descarado, te quiero, "quién es el padre de tu hijo", "estoy enamorada de ti... y muchas de ese tipo". A esto mismo apunta Marcel Gascón, ex corresponsal de EFE: "Estoy embarazada, solían decir las chicas en broma, y se reían. También cosas como te quiero José Armando, también en broma" (Marcel Gascón, comunicación personal, 7 de junio de 2013).
Luego, resulta necesario preguntarse cuánto se aprende. Por eso, se solicitó a las 21 personas describir su propio nivel de español en las cuatro habilidades, aun cuando las telenovelas impliquen únicamente la oral y la de escucha. Esta descripción se ordena en la Tabla 3. Debe tomarse en cuenta que de las 21, solamente 9 personas han llevado cursos de español y se evaluaron: 5 como avanzadas, en todas o ciertas habilidades -una solo en escucha Marcel Gascón; las otras, 2 como intermedias (1 por cómo describió sus habilidades) y 1 como principiante. 
Tabla 3: Evaluación de las habilidades aprendidas

\begin{tabular}{cccccc}
\hline Nivel/Habilidad & Escucha & Habla & Escritura & Lectura \\
\cline { 2 - 3 } Avanzado, muy bueno & 12 & 5 & & 2 & 9 \\
Medio, bueno, suficiente, bueno, aceptable & 8 & 9 & 6 & 7 \\
Principiante, débil & 1 & 5 & 7 & 4 \\
Solo algunas frases y palabras & - & 2 & 1 & - \\
No sé hacerlo, para nada & - & - & 5 & - \\
Otras respuestas: "creo que podría hacerlo" & - & - & - & 1 \\
\hline Total de personas & 21 & 21 & 21 & 21 \\
\hline
\end{tabular}

Para llegar al nivel actual, las personas afirman haber visto telenovelas durante más de un año -quienes indicaron menos tiempo-, y durante 17 años -quien indicó más-. Un dato importante es la seguridad de algunas personas con respecto a la lengua aprendida: "En el instituto Cervantes en Bucarest, no continué porque mi nivel era mucho mayor que B2, en el que fui colocada inicialmente con base en mis conocimientos gramaticales".

Esta diferencia entre el nivel de lo aprendido de las telenovelas y el requerimiento gramatical de la enseñanza reglada lleva a preguntarse si hablantes nativos de español perciben una distinción entre el español que se aprende de las telenovelas y el que se aprende por otros medios. La respuesta unánime de quienes opinan que se puede aprender es sí. Uno indica: “... primero el acento, segundo las palabras y tercero en novelas no aprenden casi gramática"; otro afirma que: “... utilizan muchos regionalismos mexicanos dato muy extraño teniendo como más próximo el país España". Para el ex corresponsal de EFE: "hay quizá una diferencia de registro: quien aprendió español con las telenovelas habla a menudo un idioma mucho más vivo y práctico que el que aprendió en las instituciones educativas" (Marcel Gascón, comunicación personal, 7 de junio de 2013).

Con respecto a la utilidad del español aprendido, algunas de las personas rumanas perciben que ha sido de gran provecho: en situaciones de trabajo (en Rumanía y España); en viajes; para escuchar y, luego, empezar a hablar con grupos de españoles. Para una informante, "lo más increíble fue cuando caí en cuenta de que con mi nivel de español acumulado del seguimiento de telenovelas, junto con solo unas cuantas lecciones autodidácticas de gramática, era capaz de obtener una beca Erasmus en España”.

Según quienes participaron como informantes de origen latinoamericano, de sus 28 a 32 conocidos que aprendieron español de las telenovelas, el número de personas con las que se puede entablar una conversación es elevado: 22 o 23. Para el ex corresponsal de EFE, "más allá de la fase cómica las chicas [...] podían desenvolverse bien en español en situaciones cotidianas e íntimas, que son las que se aparecen en las telenovelas" (Marcel Gascón, comunicación personal, 7 de junio de 2013) y "a veces incluso consiguen trabajos gracias a su aprendizaje del idioma por esas series" (Marcel Gascón, 
comunicación personal, 7 de junio de 2013). El aporte de Gascón resulta oportuno al considerar los ámbitos de las primeras palabras que las personas rumanas indicaron haber aprendido por medio de las telenovelas.

\section{De la telenovela a la lengua española}

Con las telenovelas los receptores de Rumanía consumen principalmente español americano (Tabla 1), pero el país tiene mayor contacto con España por su cercanía. Por esta razón, toma importancia la pregunta de si observan diferencias entre el español peninsular y el latinoamericano. Para conocer este dato, se realizó la pregunta a las 25 personas informantes. De ellas, 3 opinan que no y 22 opinan que sí existe diferencia. Las diferencias que mencionan fueron organizadas por tipos como se expresa en la Tabla 4:

Tabla 4: Principales diferencias entre el español peninsular y el americano según los rumanos

\begin{tabular}{llc}
\hline \multicolumn{1}{c}{ Tipo } & \multicolumn{1}{c}{ Diferencia } & N.` menciones \\
\hline Fonética & $\begin{array}{l}\text { de acento, de entonación, de pronunciación de los sonidos, } \\
\text { melodía }\end{array}$ & 18 \\
Morfosintáctica & pronombres de cortesía & 1 \\
Léxica & de expresiones, de vocabulario, de palabras, regionalismos & 11 \\
Otros & "dialectales" & 2 \\
No especificada & "no puedo explicar", "creo que me doy cuenta", "...” & 3 \\
\hline
\end{tabular}

La caracterización de las variedades dialectales no depende de si las personas han llevado cursos de lengua española o no. De las 3 personas que respondieron que no existen distinciones, una ha llevado cursos y no aprendió español por las telenovelas, y dos no han llevado cursos y aprendieron de las telenovelas. Por ejemplo, la persona que incluyó como diferencia los pronombres de cortesía no ha llevado cursos; indicó que se diferencian en el uso del tú en España y en América, del vos. A pesar de que el tú se utiliza también en América, resalta el hecho de que se haya asociado el pronombre vos con Latinoamérica, de donde sí es exclusivo. También, entre las respuestas resaltan una muy específica de una persona que llevó cursos y afirmó que hay diferencias "incluso entre los países latinoamericanos", y una más especializada de una persona que estudió español en un contexto formal (universitario) que mencionó grupos fónicos.

Al parecer, la diferenciación que hacen las personas rumanas entre ambos españoles es bastante evidente, incluso para hispanohablantes, a quienes se les preguntó primero por la opinión general que han escuchado de rumanas y rumanos acerca del español. Las personas latinoamericanas indicaron que "les gusta mucho" el español, y que 
según las personas de ese país: "es fácil de aprender y sí también es muy musical y bonito". Según uno de los entrevistados, "es un idioma bastante accesible para ellos, más que otros idiomas de Europa del Este por ejemplo”.

Luego, se preguntó a quienes provenían de Latinoamérica si las personas rumanas diferencian entre el español peninsular y el americano: 4 dijeron que sí. Uno de los informantes indica que "algunos saben incluso reconocer los diferentes acentos. Las novelas probablemente los sensibilizan a esas diferencias". Además, un informante que ha residido durante 21 años en Rumanía opina que la introducción de las telenovelas a ese país tiene relación con el conocimiento de las variedades diatópicas: “... los rumanos que aprendían español estudiaban directamente el castellano enseñado por medio de la gramática de la real academia de España [sic]; así que cuando entraron las novelas el español que nosotros hablamos es un poco diferente". También el ex corresponsal de EFE apuntó que los rumanos hacen la distinción: "Es curioso cómo -y cada vez menos porque cada vez hay más españoles de España [sic] en Rumanía- para algunas rumanas el español estándar es el de América, pues es el que ellas conocen mejor -por las telenovelas-" (Marcel Gascón, comunicación personal, 7 de junio de 2013).

Como la mayoría de los receptores percibe la variedad entre españoles, tiene posibilidad de posicionarse frente a la lengua y preferir una de las variantes; por eso, cabe la pregunta por las razones para elegir una u otra variedad. De las 22 personas rumanas que opinan que existe diferencia entre las dos regiones: 9 no tienen preferencia, 7 prefieren el de América y 6 el de España. Las razones para preferir una u otra se presentan en la Tabla 5:

Tabla 5: Razones de preferencia de una variante del español

\begin{tabular}{|c|c|c|}
\hline & Razón & N. ${ }^{\circ}$ menciones \\
\hline \multirow{4}{*}{ 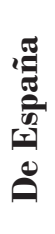 } & Corrección & 1 \\
\hline & Musicalidad (melodía, "me gusta más como suena") & 2 \\
\hline & Porque esta fue la que aprendí, porque a esta me acostumbré & 1 \\
\hline & Cercanía con el latín & 2 \\
\hline \multirow{7}{*}{ } & Accesibilidad & 1 \\
\hline & Simplicidad & 1 \\
\hline & Facilidad & 1 \\
\hline & Pronunciación más fácil & 1 \\
\hline & Porque esta fue la que aprendí & 1 \\
\hline & $\begin{array}{l}\text { Musicalidad: asociada con lo expresivo ("me parece más sonora y más } \\
\text { llena de expresividad") y lo afectivo ("me parece más calidad, más } \\
\text { dulce, más cantada") }\end{array}$ & 2 \\
\hline & No indica razones & 1 \\
\hline
\end{tabular}


Al organizar estas razones, se observa que la mayoría se declara "sin preferencia”. No obstante, ni la inclinación por una $u$ otra variable -inclusive para quienes declararon tener una- ni la neutralidad con respecto a ellas es absoluta. Por ejemplo, una de las personas que dice no tener preferencia, aclaró que: “... de todos modos voy con la de América Latina, porque he visto muchas más telenovelas de esa zona". Asimismo, una informante que estudió una maestría en español indicó que prefiere la peninsular, pero aclaró que "durante el último tiempo me he acostumbrado al español de España. Pero mi preferido de escuchar sigue siendo el español de Argentina". Finalmente, para una persona "la de América Latina es más fácil y es con la que me he acostumbrado a lo largo del tiempo. El acento de los de España es uno que, muchas veces, hace difícil la comprensión"; con este último comentario coincide Gascón: "El español de América también les resulta más fácil, dicen algunos, por la ausencia de "ces" y por hablarse a un ritmo más lento y armónico" (comunicación personal, 7 de junio de 2013).

\section{Lengua como producto}

Un posicionamiento inicial de este trabajo fue que el consumo del español por medio de las telenovelas puede motivar a quienes son receptores y receptoras a consumir español por medio de la enseñanza reglada. Esta idea no pareciera ser apoyada por los datos obtenidos. De las 21 personas que aprendieron español viendo telenovelas, solo 9 han llevado cursos y 12 no lo han hecho. De las 9 , únicamente 3 dicen que las telenovelas influyeron para tomar esos cursos. Debe sumarse que de 4 personas que no aprendieron español de las telenovelas, 1 indica que estas sí influyeron en su decisión de tomar cursos. En otras palabras, 4 de las 25 personas han tomado cursos por causa -probablemente entre otras razones- de las telenovelas. Además, vale resaltar que una persona que aprendió español viendo telenovelas indicó no solo haber llevado cursos, sino incluso haber estudiado Filología Española.

Como se evidencia, entre las personas entrevistadas, el número de receptoras que aprendió español es mucho mayor que el que se interesó en asistir a cursos. Además, sobresale el caso ya mencionado de una persona que sí llevó un curso de español, pero lo abandonó por sentir que la ubicación - de acuerdo con la gramáticano correspondía con el nivel que ella tenía a partir de las telenovelas.

A pesar del no cumplimiento de la hipótesis señalada, debe mencionarse que desde la percepción latinoamericana, las telenovelas sí juegan un papel "influyente a que las personas aprendan y se sienten interesadas por el idioma", pues implican "una idea de un mundo extraño, algo que no conocen y les gustaría conocer por el estilo de vida tan pero tan diferente al de ellos que les parece asombroso. Es como por donde pueden espiar una cultura que tanto admiran". Aun así, para dos de los hispanos, la telenovela en español en Rumanía es sobre todo diversión popular o una atracción.

Otra idea de partida fue que el consumo de telenovelas tenía relación con la preferencia de las personas receptoras por la variedad estilística americana del español; este punto tampoco es apoyado por los resultados, porque los 
grupos que no prefieren ninguna o quienes prefieren una $u$ otra variedad son semejantes en número, aunque todas las personas hayan visto telenovelas.

No obstante, en las razones expuestas por hablantes, sí se ve reflejado que en el mercado lingüístico los productos que circulan son "discursos estilísticamente caracterizados", como los define Bourdieu (2001). De este modo, estilísticamente, el español peninsular se asocia con musicalidad, cercanía al latín y corrección. Por su parte, el español americano se asocia con sencillez, accesibilidad, facilidad de pronunciación y musicalidad asociada a la expresividad y los sentimientos. La expresividad atribuida al español americano, precisamente, podría asociarse con los mensajes de las telenovelas. Sin embargo, los datos obtenidos serían insuficientes para apoyar esta afirmación.

Con respecto al posicionamiento de partida de una preferencia por el español americano, debe rescatarse la opinión de algunos hispanohablantes para quienes podrían mediar diversos factores, por ejemplo: “... a muchos rumanos no les caen bien los españoles por que [sic] los confunden con los gitanos, en cambio por los latinos sienten mucha admiración”. Este grupo también señala una posible limitación que debe considerarse en futuros trabajos sobre la difusión de la cultura de habla hispana por medio de este tipo de programas televisivos: "en general las telenovelas permiten ver algunos aspectos de la vida y de la cultura de un país. Un problema o riesgo es que también pueden generar prejuicios o una visión parcial o simplista de un país”.

\section{Conclusiones}

Los datos expuestos permiten concordar con Fiallo (citada por Covarrubias, 2010, párr.109) en que: "la telenovela es un género multitudinario [con] gran poder de penetración". Lo sorprendente de este poder es la capacidad de borrar los límites entre lenguas al llevar a las personas rumanas que consumen los productos lingüísticos de dicho género a convertirse en aprendientes inconscientes del español, como ellos lo describen. Por medio de la totalidad de las preguntas, se evidencia cómo las personas receptoras de este tipo de programa lo validan como un mecanismo para aprender de la lengua española y conocer distintas variantes del español.

Sería adecuado estudiar a fondo la actitud de la población rumana hacia los aprendientes de español por telenovela y hacia quienes lo han aprendido por otros medios. Asimismo, sería necesario indagar en la representación que tienen las personas de dicho país acerca de las variantes de España y América, y las ideologías asociadas a dichas representaciones, pues como se demostró estas dos variantes confluyen en el mercado lingüístico de dicho país. Igualmente, habría que hacer un trabajo detallado sobre los distintos españoles de América y su proyección en Rumanía y otros países donde existan aprendientes de ELE por medio de telenovelas.

Para concluir, debe considerarse que las telenovelas no siempre impulsan el interés por la enseñanza reglada del español ni la preferencia del español americano, pero sí llevan a las personas televidentes a aprender la variedad que se utiliza en ellas (la latinoamericana mayoritariamente, según 
los datos obtenidos) y ser capaces de emplearla en situaciones prácticas. De esta manera, es posible afirmar que las telenovelas contribuyen a la globalización lingüística del español latinoamericano y sus variedades. Así las cosas, es posible dar razón a quien afirmó que las telenovelas «...pueden modificar la historia futura de la lengua; los culebrones pueden hacer mucho más por el idioma castellano que, por ejemplo, una reunión de academias» (Gregorio Salvador, citado por Covarrubias, 2010, párr.80).

\section{Notas}

1. Este trabajo fue presentado en el IV Congreso Internacional de Lenguas Modernas celebrado en la Universidad de Costa Rica en diciembre de 2014.

2. También, se ha investigado sobre los medios de comunicación como motivación para el aprendizaje de español como segunda lengua, aunque específicamente en EE. UU. (Fonseca y García, 2010).

3. Se procuró contactar por correo electrónico el Instituto Cervantes de Bucarest para la obtención de datos adicionales; sin embargo, no fue posible.

4. Si bien la telenovela es el punto central para esta investigación, se tiene en cuenta que no es el único factor que genera interés por el español. Un estudio que quiera dar cuenta de la relación de los rumanos con esta lengua debe incluir otros factores -culturales, políticos y geográficos, entre otros-.

5. Para los fines de este estudio, no se toman en cuenta todas las variedades dialectales de los países de la región que son utilizadas en las telenovelas, sino que se entiende el español de
América en oposición al peninsular, más cercano geográficamente a Rumanía.

6. El interés por realizar este estudio surge durante una estancia de inmersión cultural (2009-2010) en la cual fue posible observar el fenómeno.

7. La traducción de todos los datos provenientes de informantes rumanos es propia.

\section{Bibliografía}

Bernárdez, E. (2008). El lenguaje como cultura. Madrid: Alianza Editorial.

Bourdieu, P. (2001). ¿Qué significa hablar? Madrid: Ediciones Akal.

Centro Cervantes Virtual. (s.f.). "Aprendizaje de segundas lenguas". En Diccionario de Términos clave de ELE. Recuperado el 21 de junio de 2013, de: http:/cvc.cervantes.es/ensenanza/ biblioteca_ele/diccio_ele/indice.htm

Covarrubias, J.I. (2010, marzo). Las "telenovelas ejemplares": Thalia, Betty la fea y el idioma de Cervantes. Ponencia presentada en el V Congreso Internacional de la Lengua, Valparaíso. Recuperado el 25 de abril de 2013, de: http://congresosdelalengua.es/valparaiso/ponencias/america_lengua_espanola/covarrubias_jorge_i.htm

EFE. (2009, 21 de marzo). "Miles de rumanos aprenden español con las telenovelas latinoamericanas". El Mundo. Recuperado el 25 de abril de 2013, de: http://www.elmundo. es/elmundo/2009/03/21/comunicacion/1237653895.html

Fonseca, C. y García, L. (2010). “Aprender español en USA: los medios de comunicación como motivación social". Comunicar Revista Científica de Educomunicación, N. ${ }^{\circ 34}$. Pp.145-153. 
Fuentes González, D. (2001). "Valoración y actitudes sociolingüísticas hacia el español americano". En Calvo, J. (Ed.). Contacto interlingüistico e intercultural en el mundo hispánico. Vol. 1. Valencia: Instituto Valenciano de Lengua y Cultura Amerindias. Pp-113-143.

Fuenzalida, V. (2001). "La TV como industria cultural en América Latina". Pharos, 8, 1. Pp. 3-45.

Gascón, M. (2013, 7 de junio). Comunicación personal.

Jimeno Panés, M. (2006). "El español en Rumanía y Moldavia”. En Centro Virtual Cervantes. Enciclopedia del español en el mundo. Pp. 304- 311. Recuperado el 25 de abril de 2013, de: http://cvc.cervantes.es/ lengua/anuario/anuario_06-07/pdf/ paises_66.pdf

López Morales, H. (2006). La globalización del léxico hispánico. Madrid: Espasa.

Mazziotti, N. (2006). Telenovela: industria y prácticas culturales. Bogotá: Grupo Editorial Norma.

Oproiu, D. (2012, 10 de setiembre). "Elena de la Mandinga: P'Am învatat spaniola datorita telenovelelor/'. Evz. ro. Recuperado el 26 de abril de 2013, de: http://www.evz.ro/detalii/stiri/Elena-de-la-Mandinga-Am-nvat-spanioldatorit-telenovelelor-1000083.html

Ponzio, A. (2008). Linguaggio, lavoro e mercato globale: rileggendo Rossi-Landi. Milán: Mimesis. Recuperado el 16 de junio de 2013, de: http:// www.ferrucciorossilandi.com/files/ ponzio_11.pdf

Rossi-Landi, F. (1970). El lenguaje como trabajo y como mercado. Caracas: Monte Ávila Editores.

Salgueiro, R. 2004. "Televisión/Europa del Este en romance con la telenovela latinoamericana". Revista Latinoamericana de Comunicación CHASQUI, número 87. Pp.66-71.

Tucan, G. 2007, 29 de octubre. "Andrea Antonescu invata spaniola din telenovela". 9AM News. Recuperado el 24 de mayo de 2013, de: http://www.9am. ro/stiri-revista-presei/2007-10-29/andreea-antonescu-invata-spaniola-dintelenovele.htmly 
